\title{
NUMERICAL COMPARISON OF TRANSGINGIVAL AND SUBGINGIVAL DENTAL IMPLANTS IN REGARD TO THEIR STRESS DISTRIBUTIONS
}

\author{
Luboš ŘehouneK ${ }^{a, *}$, Aleš Jíra ${ }^{a}$, Gabriela Javorská ${ }^{a}$, DANiel Bodlák ${ }^{b}$ \\ a Czech Technical University in Prague, Faculty of Civil Engineering, Department of Mechanics, Thákurova 7, \\ 16629 Prague 6, Czech Republic \\ ${ }^{b}$ ProSpon spol. s.r.o., Jiřího Voskovce 3206, 27201 Kladno, Czech Republic \\ * corresponding author: lubos.rehounek@fsv.cvut.cz
}

\begin{abstract}
Most modern dental implants differentiate in regard to the fixation of the abutment into two main categories - the external or internal hexagon or octagon. We performed mechanical tests according to the ČSN EN ISO 14801 standard on a dental implant variant using the external hex. We found that failure of all implant specimens occured below the screw head. To improve the current geometry, we performed numerical analysis of an alternative variant (internal hex) and compared it with analysis of the current geometry (external hex). It was found that the stress distribution of the variant with internal hex is preferable to the old variant. Although extreme values of shear stress in the corresponding plane of loading are higher, they do not concentrate below the screw head, where the screw itself is thinner and more prone to breaking. Therefore, it seems that the new variant of the dental implant is stronger, which is still to be proven by mechanical tests.
\end{abstract}

KEYWORDS: External hexagon, FEM, implant-abutment interface, internal hexagon, stress.

\section{INTRODUCTION}

The implant-abutment connection is one of the most important interfaces of a dental implant. It provides a means to apply torque required to screw the implant into bone and also serves as a connection between the intraosseous part and the abutment. There are many requirements and functions of this interface that may be less or more propagated in regard to the form of connection (internal or external). However, one of the most important functions is the prevention of rotation between individual parts [1]. This particular property is greatly influenced by the character of the interface.

From a mechanical standpoint, there are several geometrical solutions among dental implants in regard to their placement and the fixation of the abutment. They include the Internal Morse Taper, External Morse Taper, internal lobing, tube-to-tube systems and external and internal polygons. These last 2 main categories comprise the vast majority of clinically used implants as well as the majority of market share. The most common variants of the polygonal fixation are the hexagon or the octagon. Our study includes 2 different variants of the polygonal variant the external hexagon (transgingival variant) and the internal hexagon (subgingival variant). Their names suggest their placement in the bone, as shown in Fig. 1 .

Historically, the first manufactured titanium dental implants by Brånemark had the external hexagonal connection. It is a variant that is very simple a robust, however, it has some deficiencies, such as insufficient anti-rotational resistance or insufficient resis-

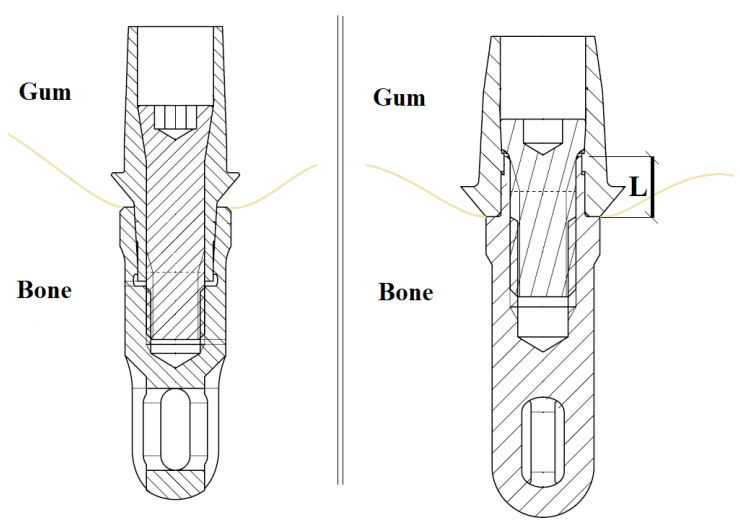

Figure 1. Comparison of placement of the 2 analysed dental implant variants inside the bone. Left the internal hexagon variant (subgingival placement) and right - the external hexagon variant (transgingival placemenent). The lenght $L$ indicates the portion of the intraosseous part that exceeds the bone after implantation.

tance to intraoral forces with greater offset from the connection (e.g. top of the crown). To improve lateral and rotational stability, many other variants were developed (internal hex, spline connection, Morse Taper, tapered hexagon and other variants) [2].

\subsection{Motivation FOR DEVElopment}

The literature suggests that internal connections have mechanical advantages. It was found that the bending moments of zirconia implants are superior when paired with internal connection, compared to exter- 


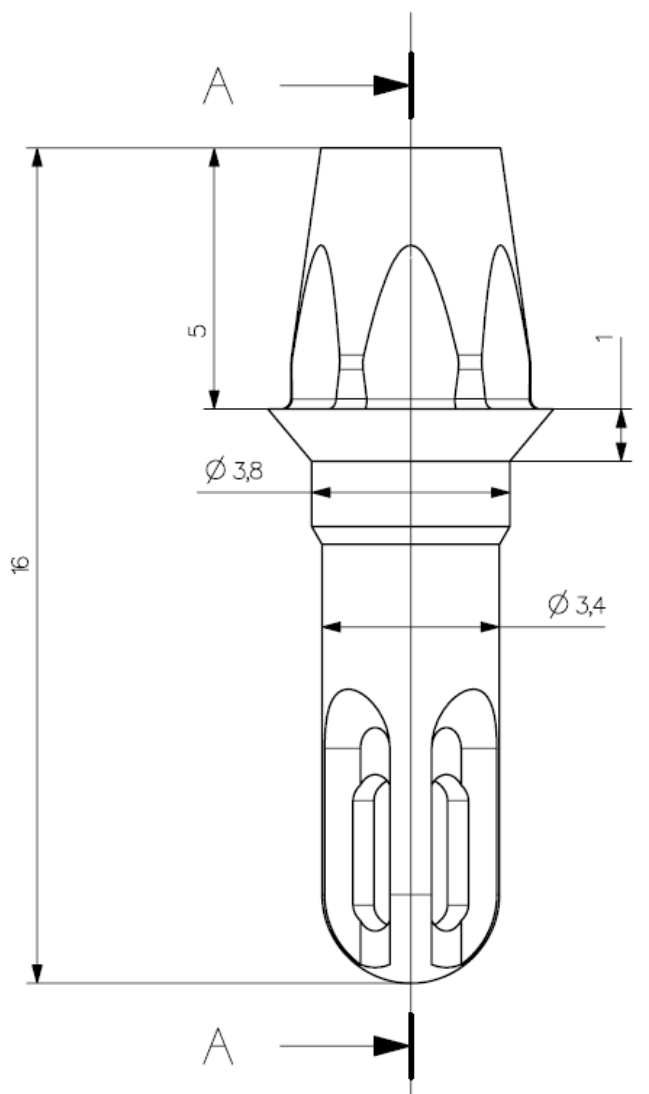

Figure 2. Documentation of the old external (EH) connection variant of the implant.The intraosseous part is the subject of a patent [7].

nally connected abutments [3, 4]. Research suggests that external connections lead to greater stresses inside the components of the implant, mainly at the connection interface [5]. Also, they pose as an alternative to external connections, which have proven to break in the abutment screw [6]. The abutment screw can be identified as a very weak component of the system as it is thin while being exposed to shear forces during mastication.

The motivation to develop other variants of connection also stems from the fact that external connections have proven to decrease levels of crestal bone at the peri-implant area after 1 year of loading and also exhibit greater bone remodelling and bone loss when compared to internal connections within the same set of circumstances [8]. Furthermore, internal abutment connections have better lateral stability as the lever arm of forces is shorter, thus reducing the value of maximum force that can be applied in the lateral direction. According to literature, this results in better stress distributions, less micromovement and higher survival probability [9]. Other factors, such as surface treatment, anodization, implant length, abutment material etc. also influence bone loss.

Another aspect of potential improvement over the external connection types is improving the transfer of loads in the surrounding bone and minimizing the gaps at the implant-abutment interface which may

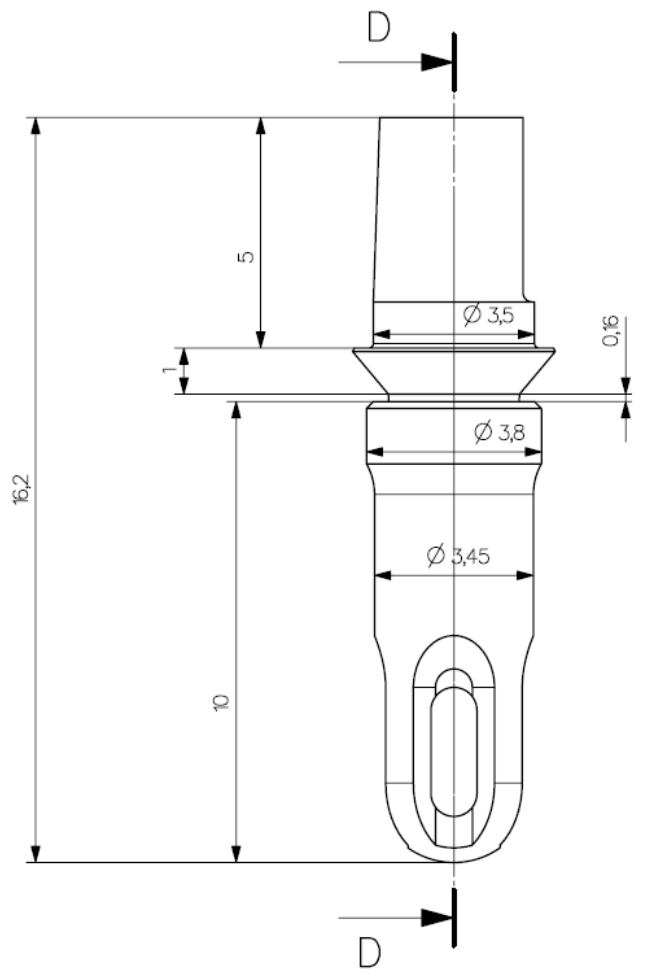

Figure 3. Documentation of the new internal (IH) connection variant of the implant.The intraosseous part is the subject of a patent [7].

lead to bacterial colonization [10] and subsequent biofilm accumulation [11].

\section{Materials And Methods}

The means of comparison in this study are FEM analyses of 2 selected dental implant variants in regard to their stress distribution. The selected variants are the external hexagon $(\mathrm{EH})$ and the internal hexagon (IH), as shown in Fig. 2 and Fig. 3. The simulations were performed on both variants on the basis of conditions and results of mechanical tests of the EH variant so as to verify the viability of the newer $\mathrm{IH}$ variant in regard to stress distribution.

\subsection{Mechanical Tests}

We performed mechanical tests on the old EH variant according to the ISO 14801 Dentistry-ImplantsDynamic fatigue tests for endosseous dental implants standard [12] on a total of 6 specimens of dental implants (Fig. 4). The tests were performed on a double-column pneumatic press LiTeM Vertical Double Column VDC A-6. The machine complies with ISO 7500-1 [13] and enables for constant crosshead displacement.

A sample P-D diagram of the tested EH specimens is shown in Fig. 6. All specimens were embedded in the Dentacryl fixating medium (methylacrylate resin for technical use). The resin has good mechanical properties and thermal insulation properties. Its bending strength is $50 \mathrm{MPa}$, tensile strength is 20 


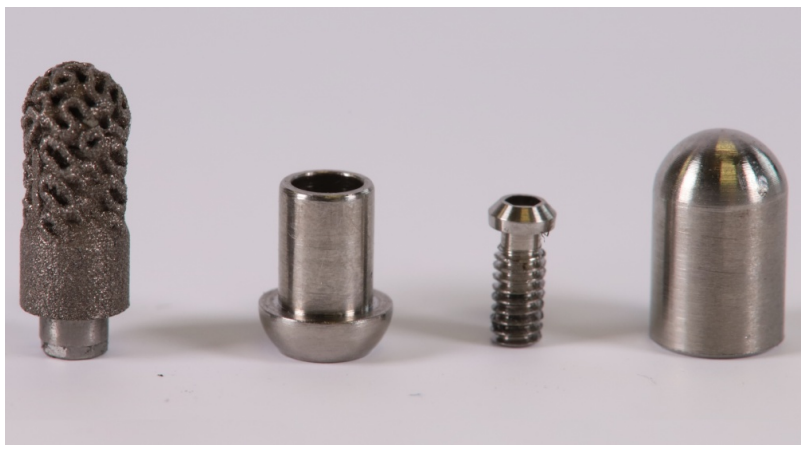

Figure 4. A disassembled specimen of the EH variant used for the mechanical tests. Although the body of the implant is different, the locking system including the abutment and screw are the same.

\begin{tabular}{cc}
\hline Specimen Number & Force $[\mathrm{N}]$ \\
\hline 1 & 1278.7 \\
2 & 1387.6 \\
3 & 1301.2 \\
4 & 1486.0 \\
5 & 1275.8 \\
6 & 1233.2 \\
Mean Value & 1327.1 \\
\hline
\end{tabular}

TABLE 1. Maximum static load force for all EH specimens.

$\mathrm{MPa}$ and compressive strength is $80 \mathrm{MPa}$. Its elastic modulus is $3.2 \mathrm{GPa}$. With these properties, it complies with the mentioned standards for static testing.

As the ISO 14801 standard [12] requires a $30^{\circ}$ slanted force, a fixation apparatus is needed to slant the specimens as the LiTeM machine can only apply force in the vertical direction. We needed to make this preparation ourselves as the manufacturer does not provide any further preparations for the machine. It was modelled in NetFabb and subsequently 3D printed on the Sinterit Lisa Pro 3D printer by using the SLS (Selective Laser Sintering) technology. The used material was the PA12 polyamide, whose strength is comparable with aluminum. The fixating preparation is shown in Fig. 7.

After the fixation, the implant is connected with the abutment by the abutment screw with a M2.2 thread using a $250 \mathrm{Nm}$ momentum by a special ratchet supplied by the manufacturer of application instrumentation. The mechanical tests were controlled by a constant speed of displacement of 4 $\mathrm{mm} / \mathrm{min}$ until specimen failure in the LiTeM machine.

The mean maximum force of the implant specimens at failure was $1327.1 \mathrm{~N}$ (Table 1) and the failure occured below the head of the abutment screw for all EH specimens, as shown in Fig. 5. This test confirmed the hypotheses that the abutment screw would be the weakest part of the assembly and also corrobo-

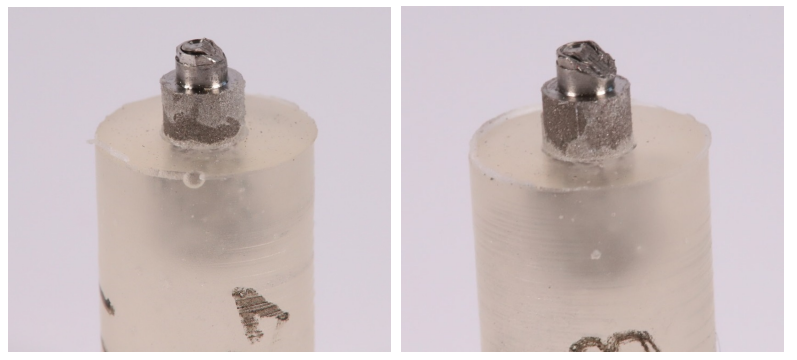

FiguRE 5. Two EH specimens after mechanical tests. The images show failure of the specimens right below the head of the abutment screw. Note that all specimens failed exactly in this manner.

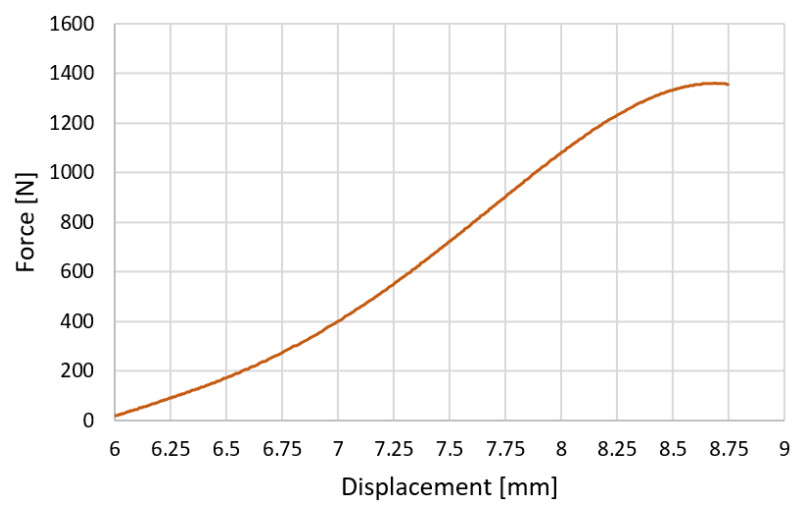

FiguRE 6. A sample P-D diagram of specimen n. 2 from the mechanical testing. The maximum force for this specimen was $1387.6 \mathrm{~N}$.

rated the research of [6]. From Fig. 5, shear failure of all specimens is apparent. The failure of all implants occured by shear failure of the screw connecting the intraosseous part and the abutment. This connection proved to be a limiting factor for the tested EH specimens. A positive finding was the fact that the bodies of the implants themselves remained intact.

\subsection{FEM ANALYSES}

As all of the EH specimens achieved failure below the head of the abutment screw, we set out to model these results using ANSYS Workbench $2020 \mathrm{R} 1$. The overall approach was to model the older $\mathrm{EH}$ variant, verify the model by comparing it with mechanical tests and subsequently analyse a newer version of the implant with an internal hexagonal connection to confirm whether distributions of stresses are better.

The geometrical models were imported into ANSYS in the form of a parasolid (.x_t extension) and are shown in Fig. 9. According to the previously conducted mechanical experiments, we chose a force with the magnitude of $900 \mathrm{~N}$ as it is approximately the highest force that is still in the linear region of the P-D diagram (Fig. 6). The force is situated on top of the abutment rim and distributed equally among a few nodes of the mesh (Fig. 9). To accurately represent the conditions of the experiment and the standard [12], we fixed the lower part of the implant that was embedded in the Dentacryl resin and left 


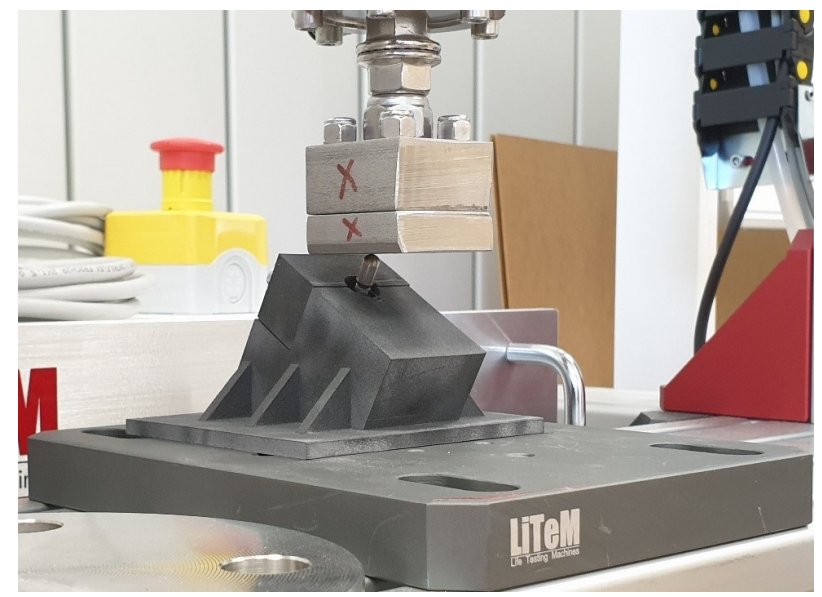

Figure 7 . The mechanical testing assembly with the LiTeM machine and a 3D-printed preparation for anchoring the specimens at a $30^{\circ}$ angle.

a $2 \mathrm{~mm}$ gap between the top of the fixed area and the theoretical level of bone (Fig. 9). As the goal of the simulation was to compare stress distributions during the linear behavior, we did not consider nonlinear behavior in the model and chose the same force of $900 \mathrm{~N}$ for both simulations.

Simple tetrahedral linear elements and linear material behavior with using the Ti-6Al-4V material model were used to model the numerical problem. The mesh was designed so as to represent at least 5 elements across the width of the stabilizing ribs of the implant substructure and was further refined by size in the region of interest - the abutment screw.

As the assembly consists of 3 solids - the abutment, the screw and the intraosseous part - the model contains basic contact conditions with specific distance break criteria. The contact conditions are necessary as some parts have conical shapes, e.g. the fit is assured by screwing the screw into the abutment conical cavity, the screw passing through the intraosseous part of the implant etc. The contact conditions are shown in Fig. 8.

Due to the complicated geometry of the real screw, we decided to only model its threaded body as a cylinder as the goal of the simulation was not to observe stress distributions in individual threads or details, but rather stress distributions across the whole screw body, i.e. compare the stress concentrations or lack thereof.

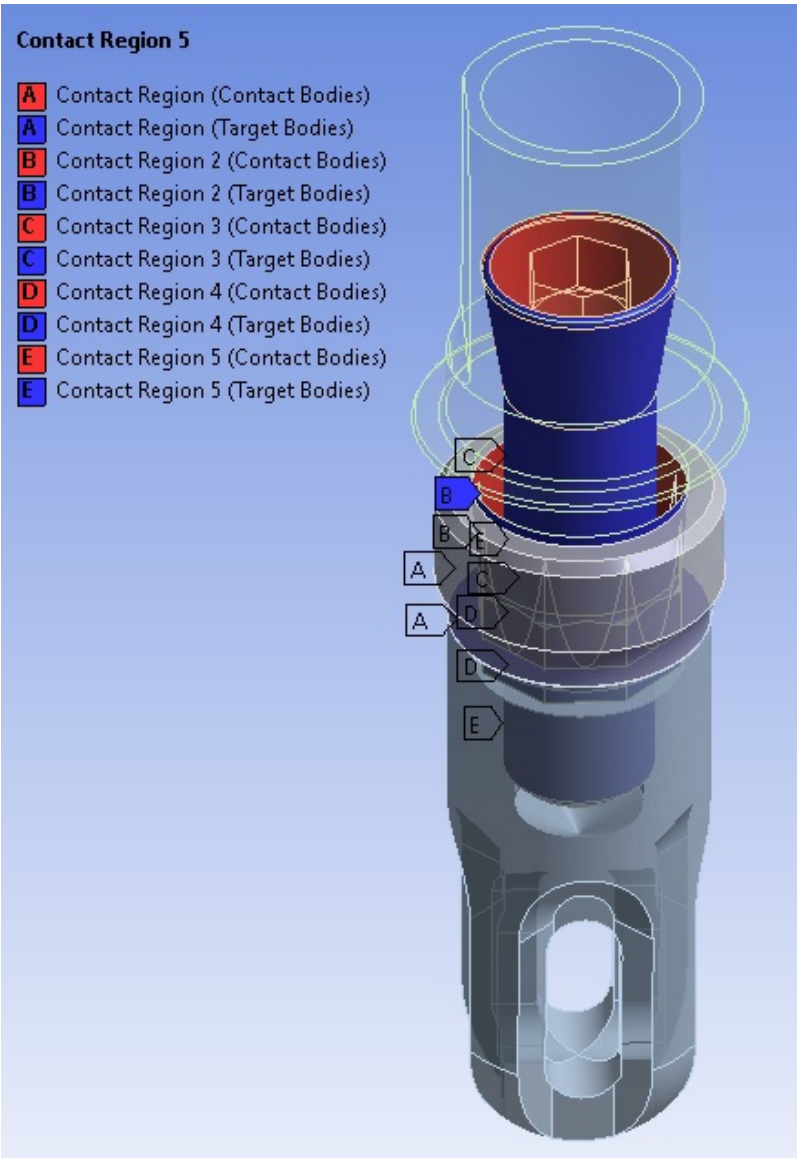

Figure 8. Contact regions of the new IH variant inside the ANSYS environment.
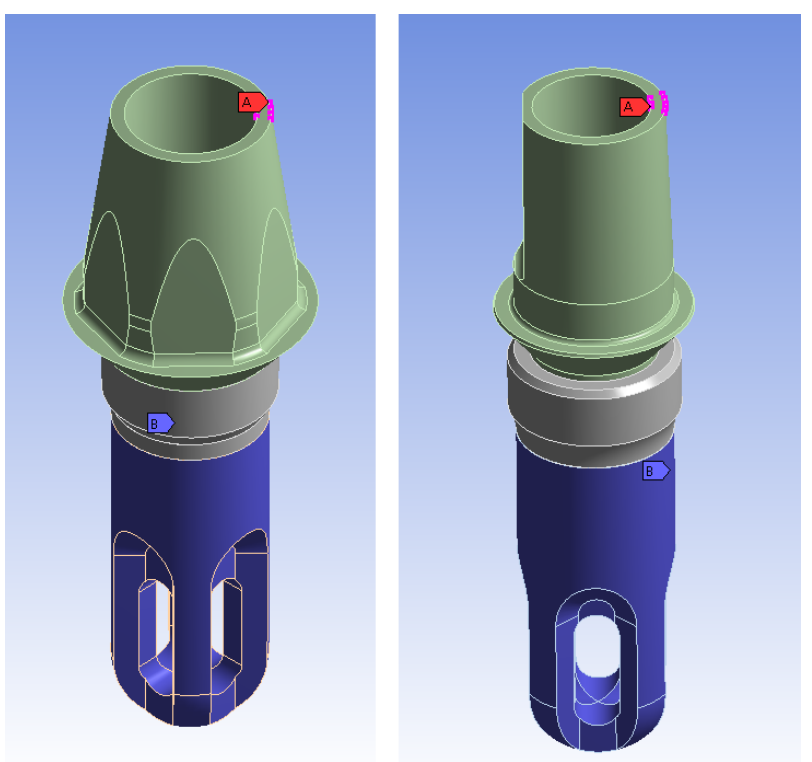

Figure 9. The two geometrical models inside the ANSYS environment. Left- the old EH variant. Right - the new, IH variant. Blue shows the area of fixation (2 $\mathrm{mm}$ below the theoretical level of bone), red shows the location of the $30^{\circ}$ slanted force. Boundary conditions comply with previous mechanical tests and with ISO 14801 [12]. 


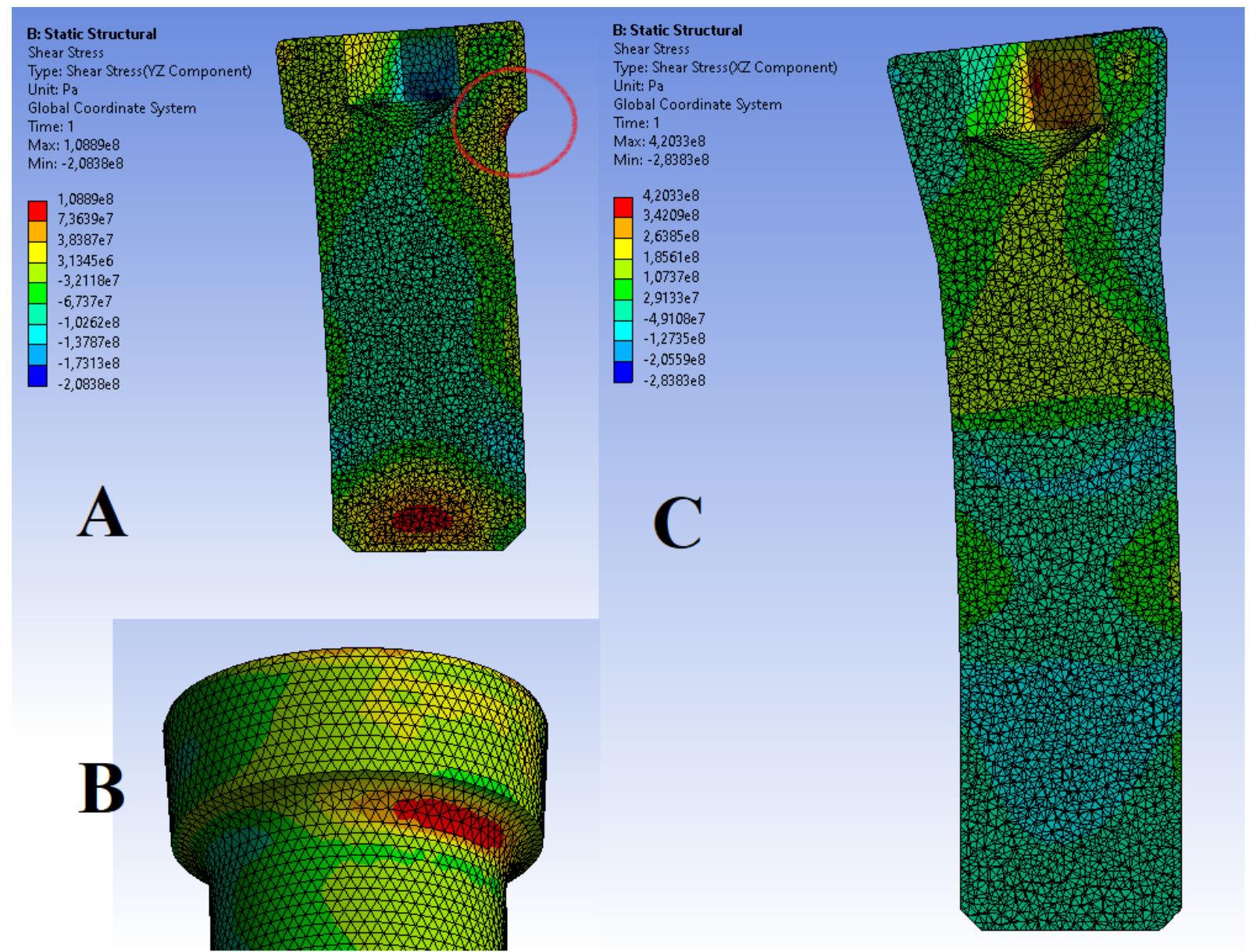

FIGURE 10. The final results of both analyses (EH and IH variant) of the FEM analyses displayed on the abutment screws. A - section through the abutment screw of the EH variant, typical stress concentration marked red. B a detail of the area highlighted in "A". C - the new IH geometrical solution with significantly reduced shear stress concentrations.

\section{Results}

The initial hypothesis was that by introducing a modified geometry of the implant-abutment connection, we can reduce the shear stress concentrations and therefore either eliminate stress concentrations or increase the maximum force at which the screw will eventually break (for the $\mathrm{EH}$ variant, the mean force was $1327.1 \mathrm{~N}$, see Table 1).

The results from the simulation of the old EH variant are shown in Fig. 10-A and B. The image shows that the model behaves in the same way as the specimens in mechanical tests. Although we did not encompass the region of failure as our analysis is only linear, it is apparent that the concentration of shear stresses occur exactly at the same place - the thin cross-section of the abutment screw below its head. With this information, we constructed the second model in the exact same way and compared the analyses. Moreover, another region with significant stress concentration emerged at the bottom of the abutment screw. However, this occurrence was not accounted for as it did not have any direct impact during the mechanical tests.

The results from the simulation of the new IH vari- ant are shown in Fig. 10-C. The shear stress concentrations were diminished and extremes moved from the thin cross-section of the screw into its head. Although the overall values of shear stresses are higher across the whole body of the abutment screw, the concentration points were eliminated by incorporating the internal connection.

\section{Conclusions}

The initial hypothesis of this experiment was that by replacing the external hexagonal $(\mathrm{EH})$ connection with internal $(\mathrm{IH})$ connection, we can eliminate stress concentrations that emerge with the use of EH because of its geometry. The mechanical tests have shown that all of the dental implant specimens reached failure during the tests by shear failure of the screw below the its head. Von-Mises criteria and principal stresses were also considered, but their results were not included in this study as their evaluation has proven to be non-essential for confirming the hypothesis.

The numerical analyses have shown that the EH variant induces concentrations of shear stress in the screw below its head. Under the same conditions, we 
performed analysis of the IH variant and found a more viable distribution of shear stress with no apparent concentrations in the body of the abutment screw.

Therefore, this analysis confirms our initial hypothesis that the internal connection might be more favorable and corroborated the results provided by literature [6]. The trend to innovate in the area of implantabutment connections can be seen in the market share of dental implants, where more and more companies offer alternatives to the tried-and-true external connection, which proves to have its shortcomings, such as low anti-rotational resistance, bone loss at the peri-implant area, lower lateral stability, microgaps at the abutment-implant interface leading to bacteria colonisation and biofilm formation, etc.

To further prove if this concept is true and finish the research, we need to manufacture specimens of this new geometrical solution with internal connection and subject them to mechanical tests to determine whether the character of failure changes or the maximum force required to break the abutment screw increases.

\section{ACKNOWLEDGEMENTS}

The financial support provided by the by the Faculty of Civil Engineering, CTU, Prague, project n. SGS20/155/OHK1/3T/11 is gratefully acknowledged.

\section{REFERENCES}

[1] L. Gaviria, J. P. Salcido, T. Guda, J. L. Ong. Current trends in dental implants. Journal of the Korean Association of Oral and Maxillofacial Surgeons 40(2):50-60, 2014.

[2] D. Prithviraj, N. Muley, V. Gupta. The evolution of external and internal implant-abutment connections: A review. International Dental Research 2(2):37-42, 2012.

[3] T. C. Truninger, B. Stawarczyk, C. R. Leutert, et al. Bending moments of zirconia and titanium abutments with internal and external implant-abutment connections after aging and chewing simulation. Clinical oral implants research 23(1):12-18, 2012.

[4] I. Sailer, T. Sailer, B. Stawarczyk, et al. In vitro study of the influence of the type of connection on the fracture load of zirconia abutments with internal and external implant-abutment connections. Int J Oral Maxillofac Implants 24(5):850-858, 2009.
[5] P. Asvanund, S. M. Morgano. Photoelastic stress analysis of external versus internal implant-abutment connections. The Journal of prosthetic dentistry 106(4):266-271, 2011.

[6] W. Att, S. Kurun, T. Gerds, J. Strub. Fracture resistance of single-tooth implant-supported all-ceramic restorations after exposure to the artificial mouth. Journal of oral rehabilitation 33(5):380-386, 2006.

[7] F. Denk, F. Denk, A. Jíra. A dental implant shaft, 2017.

[8] K.-T. Koo, E.-J. Lee, J.-Y. Kim, et al. The effect of internal versus external abutment connection modes on crestal bone changes around dental implants: a radiographic analysis. Journal of periodontology 83(9):1104-1109, 2012.

[9] C. A. A. Lemos, F. R. Verri, E. A. Bonfante, et al. Comparison of external and internal implant-abutment connections for implant supported prostheses. a systematic review and meta-analysis. Journal of Dentistry 70:14-22, 2018.

[10] R. Caricasulo, L. Malchiodi, P. Ghensi, et al. The influence of implant-abutment connection to peri-implant bone loss: A systematic review and meta-analysis. Clinical Implant Dentistry and Related Research 20(4):653-664, 2018.

[11] C. Alves de Sousa, J. J. Conforte, K. S. Caiaffa, et al. Sealing agent reduces formation of single and dualspecies biofilms of candida albicans and enterococcus faecalis on screw joints at the abutment/implant interface. PloS one 14(10):e0223148, 2019.

[12] S. IOf. International standard iso 14801-dentistry-implants-dynamic fatigue test for endosseous dental implants. Geneva: International Organization for Standardization 2007.

[13] E. ISO. 7500-1. cen en iso 7500-1 metallic materialsverification of static uniaxial testing machines-part 1: Tension/compression testing machines-verification and calibration of the force-measuring system. sl: European committee for standardization, august de 2004. Document Information . 